\title{
A CLASSIFICATION THEOREM FOR ABELIAN $p$-GROUPS
}

\author{
BY
}

\author{
R. B. WARFIELD, JR.(1)
}

\begin{abstract}
A new class of Abelian p-groups, called $S$-groups, is studied, and the groups in this class are classified in terms of cardinal invariants. The class of $S$-groups includes Nunke's totally projective $p$-groups. The invariants consist of the Ulm invariants (which Hill has shown can be used to classify the totally projective groups) together with a new sequence of invariants indexed by limit ordinals which are not cofinal with $\omega$. The paper includes a fairly complete discussion of dense isotype subgroups of totally projective $p$-groups, including necessary and sufficient conditions for two of them to be congruent under the action of an automorphism of the group. It also includes an extension of Ulm's theorem to a class of mixed modules over a discrete valuation ring.
\end{abstract}

The work reported here was partly announced in [21]. To study the $S$ groups, it will be necessary to prove a classification theorem for certain modules over the ring $Z_{p}$ of integers localized at $p$. We will therefore regard $p$-groups as torsion modules over this ring, and all modules in this paper will be modules over the ring $Z_{p}$. The ring of $p$-adic integers could have been used just as well. The results are valid for modules over any discrete valuation ring. If $A$ is any $Z_{p}$-module, we define $p^{\alpha} A$ for any ordinal $\alpha$ by $p^{\alpha+1} A=p\left(p^{\alpha} A\right)$, and, if $\alpha$ is a limit ordinal, $p^{\alpha} A=\bigcap_{\beta<\alpha} p^{\beta} A$. A module is reduced if, for some ordinal $\alpha, p^{\alpha} A=0$. The smallest ordinal $\alpha$ such that $p^{\alpha} A=0$ is called the length of $A . A[p]$ is the submodule of $A$ generated by the elements of order $p$. We define

$$
U_{\alpha}(A)=\left(p^{\alpha} A\right)[p] /\left(p^{\alpha+1} A\right)[p] \quad(\alpha \geqslant 0)
$$

and let $f(\alpha, A)=\operatorname{dim}\left(U_{\alpha}(A)\right)$, where the dimension is taken over the field $Z / p Z$. The cardinals $f(\alpha, A)$ are the Ulm invariants of $A$.

If $A$ is a $Z_{p}$-module, a submodule $H$ is isotype in $A$ if, for all ordinals $\alpha$, $p^{\alpha} H=H \cap p^{\alpha} A$. If $\lambda$ is an ordinal, $H$ is $\lambda$-dense in $A$ if, for all $\alpha<\lambda, A=$ $H+p^{\alpha} A$. If $\lambda$ is a limit ordinal, $\lambda$ is cofinal with $\omega$ if there is a sequence $\lambda_{i}$,

Received by the editors September 24, 1973.

AMS (MOS) subject classifications (1970). Primary 20K10; Secondary 20K30, $20 \mathrm{~K} 40$.

Key words and phrases. Ulm's theorem, totally projective groups, isotype subgroups, invariants, cotorsion groups, automorphisms of Abelian p-groups.

(1) Research supported in part by N. S. F. grant GP-28946. 
$0<i<\omega$, of smaller ordinals, such that $\lambda$ is the supremum of the $\lambda_{i}$. We can now state a theorem which summarizes our results on $\lambda$-dense, isotype subgroups of totally projective groups.

THEOREM A. Let $G$ be a totally projective $p$-group, $\lambda$ a limit ordinal such that $p^{\lambda} G=0$, and $H$ and $K \lambda$-dense, isotype subgroups of $G$.

(i) If $\operatorname{dim}((G / H)[p])=\operatorname{dim}((G / K)[p])$ then there is an automorphism of $G$ which takes $H$ isomorphically onto $K$.

(ii) If $\lambda$ is cofinal with $\omega, H$ and $K$ are isomorphic to $G$.

(iii) If $\lambda$ is not cofinal with $\omega$, then $H$ and $K$ are isomorphic if and only if $\operatorname{dim}((G / H)[p])=\operatorname{dim}((G / K)[p])$.

The proof of Theorem A is contained in 1.6 and 3.1 below. It is a consequence of (iii) above that the groups arising in this way are not necessarily totally projective. They are all contained in a larger class, which we call $S$-groups. To study the $S$-groups, we first must study a class of mixed $Z_{p}$-modules. We give a rather unsatisfactory treatment of these modules, which has the virtue of being brief and of containing all that is needed for the application to $p$-groups. For a more intrinsic treatment, we refer to [22] and a subsequent paper.

Definition [22]. If $\lambda$ is a limit ordinal, we say a module $M$ is a $\lambda$-elementary $K T$-module if $p^{\lambda} M \cong Z_{p}$ and $M / p^{\lambda} M$ is torsion and totally projective. A module is a $K T$-module if it is a direct sum of a totally projective $p$-group and $\lambda$ elementary $K T$-modules for various $\lambda$.

To state the form of Ulm's theorem that holds for $K T$-modules, we need a new invariant, introduced in [22]. If $M$ is a module, we let $h(0, M)=$ $\operatorname{dim}(M /(p M+T))$ where $T$ is the torsion submodule of $M$. Note that if $M$ is a direct sum of cyclic modules, then $h(0, M)$ is the number of infinite cyclic summands in a decomposition of $M$. More generally, $h(0, M)$ is the number of infinite cyclic summands in a basic submodule of $M$ [11, Lemma 21]. For any limit ordinal $\lambda$, we define $h(\lambda, M)=h\left(0, p^{\lambda} M\right)$. If $M$ is a $K T$-module, it is clear that $h(\lambda, M)$ is the number of $\lambda$-elementary summands in a decomposition of $M$.

THEOREM K. The class $K$ of KT-modules has the following properties:

(i) $A$ module isomorphic to a module in $K$ is in $K$.

(ii) $A$ direct sum of modules in $K$ is in $K$.

(iii) If $M$ is a module (over the ring $Z_{p}$ ) and $\alpha$ an ordinal, then $M$ is in $K$ if erties (i), (ii), and (iii), then $C$ contains $K$.

(iv) If $C$ is any class of modules containing $Z_{p}$ and having the closure propperties (i), (ii), and (iii), then $C$ contains $K$.

(v) If $A$ and $B$ are in $K$ and $f(\alpha, A)=f(\alpha, B)$ for all ordinals $\alpha$, and 
$h(\lambda, A)=h(\lambda, B)$ for all limit ordinals $\lambda$, then $A \cong B$.

This theorem is proved in $1.3,4.6$, and 5.1 below. The $K T$-modules possess a number of other nice properties [22], which will be proved in a subsequent paper.

Definition. A $p$-group is an $S$-group if it is isomorphic to the torsion submodule of a $K T$-module. It is a $\lambda$-elementary $S$-group if it is the torsion submodule of a $\lambda$-elementary $K T$-module.

In $\S 2$ we will define a family of functors $K_{\lambda}$, where $\lambda$ is any ordinal. $K_{\lambda}$ will associate with any module a $Z / p Z$-vector space. If $\lambda$ is a limit ordinal which is not cofinal with $\omega$, we let $k(\lambda, M)=\operatorname{dim}\left(K_{\lambda}(M)\right)$. If $G$ is an $S$-group, we will show that $G$ is a direct sum of a totally projective group and $\gamma$-elementary $S$ groups for various limit ordinals $\gamma$ which are not cofinal with $\omega$, and that $k(\lambda, G)$ is the number of $\lambda$-elementary summands in such a representation.

The invariants $k(\lambda, G)$ and the Ulm invariants will classify the $S$-groups. To obtain a result for $S$-groups analogous to Theorem $\mathrm{K}$, we will need a stronger closure property to get an analogue of condition (iv) of that theorem. We say a submodule $H$ of a $Z_{p}$-module $M$ is $\lambda$-high if $H \cap p^{\lambda} M=0$, and $H$ is maximal with respect to this property.

THEOREM S. The class $S$ of S-groups has the following properties:

(i) $A$ group isomorphic to a group in $S$ is in $S$.

(ii) $A$ direct sum of groups in $S$ is in $S$.

(iii) If $G$ is a p-group and $\alpha$ an ordinal, then $G$ is in $S$ if and only if $p^{\alpha} G$ and $G / p^{\alpha} G$ are in $S$.

(iv) If $G$ is in $S$ and $\lambda$ is a limit ordinal, and $H$ is a $\lambda$-high subgroup of $G$, then $H$ is in $S$.

(v) If $C$ is any class of p-groups containing $Z / p Z$ and satisfying properties (i), (ii), (iii), and (iv) above, then $C$ contains $S$.

(vi) If $A$ and $B$ are in $S$ and $f(\alpha, A)=f(\alpha, B)$ for every ordinal $\alpha$ and $k(\lambda, A)=k(\lambda, B)$ for every limit ordinal $\lambda$ which is not cofinal with $\omega$, then $A \cong B$.

(vii) If $G$ is in $S$ then $G$ is totally projective if and only if for every limit ordinal $\lambda$ which is not cofinal with $\omega, k(\lambda, G)=0$.

This theorem is an immediate consequence of the definition, and Theorems $3.2,5.2$, and 5.3 below.

A historical remark is in order concerning the form of Theorems $\mathrm{K}$ and $\mathrm{S}$. Nunke introduced totally projective $p$-groups in [16] in order to give a homological description of direct sums of countable $p$-groups. It was $\mathrm{E}$. Walker who sug- 
gested that Ulm's theorem might generalize to this class of groups. Walker showed that if this could be done, then the class of totally projective $p$-groups would be the smallest class containing $Z / p Z$ and satisfying conditions (i), (ii) and (iii) of Theorem S. Part (iv) of Theorem $\mathrm{K}$ and part (v) of Theorem $S$ were motivated by this example. In [19], Parker and Walker proved Ulm's theorem for totally projective $p$-groups of length less than $\Omega \omega$, thus giving the first major extension of the theory of $p$-groups to groups of uncountable length. The complete proof of Ulm's theorem for totally projective $p$-groups required a different technique and an entirely new characterization of these groups, and this was proved by $P$. Hill (unpublished). A short proof (using Hill's basic ideas) has recently been given by Walker [21].

The theory presented in this paper is still very incomplete. In fact, the results open a veritable Pandora's box of unsolved and, apparently, difficult problems. $\S 6$ of this paper contains a list of ten of these.

$\S 1$ below uses Hill's methods to classify $K T$-modules and to construct certain automorphisms for totally projective $p$-groups. The second section reviews the theory of cotorsion envelopes and defines the functors $K_{\lambda}$. In $\S 3$ we classify $S$-groups and dense isotype subgroups of totally projective groups. In $\S 4$, we determine what values the invariants of a $K T$-module or $S$-group can have. This includes a new proof of the corresponding result for totally projective $p$-groups, and a proof that any subgroup of a totally projective $p$-group has the Ulm invariants of some totally projective $p$-group. $\$ 5$ completes the proof of Theorems $\mathrm{K}$ and S.

1. Extending isomorphisms and constructing automorphisms. If $G$ is an Abelian group, $H$ a subgroup and $\phi: G \rightarrow G / H$ the natural map, we say $H$ is a $p$-nice subgroup if, for all ordinals $\alpha, p^{\alpha}(G / H)=\phi\left(p^{\alpha} G\right)$.

We remark that if $G$ is a group, $p^{\alpha} G$ is a $p$-nice subgroup; and if, for all $i \in I, H_{i}$ is a $p$-nice subgroup of $G_{i}$, then $\bigoplus_{i \in I} H_{i}$ is $p$-nice in $\bigoplus_{i \in I} G_{i}$. These are the only cases we will need to prove our theorems.

We follow E. A. Walker [21] in using Hill's analysis of totally projective $p$ groups as the definition of these groups.

Definition. If $G$ is a reduced $p$-group, $G$ is totally projective if it has a family $C$ of subgroups such that

(i) If $H_{i} \in C, i \in I$, then $\Sigma_{i \in I} H_{i} \in C$,

(ii) If $H \in C$, and $X$ is a countable subset of $G$, there is a subgroup $H^{\prime} \in C$ such that $H \subseteq H^{\prime}, X \subseteq H^{\prime}$ and $H^{\prime} / H$ is countable.

(iii) $\{0\} \in C$.

(iv) The elements of $C$ are $p$-nice subgroups of $G$. 
We now summarize the known results in several theorems.

1.1. THEOREM ([7], [19], [21]). Let $T$ be the class of totally projective $p$ groups.

(i) A group isomorphic to a group in $T$ is in $T$.

(ii) $A$ direct sum of groups in $T$ is in $T$.

(iii) If $G$ is a p-group, and $\alpha$ an ordinal, then $G \in T$ if and only if $p^{\alpha} G \in$ $T$ and $G / p^{\alpha} G \in T$.

(iv) If $C$ is any class of p-groups containing $Z / p Z$ and having properties (i), (ii) and (iii), then $C \supseteq T$.

(v) If $A$ and $B$ are in $T$, and $f(\alpha, A)=f(\alpha, B)$ for all ordinals $\alpha$, then $A \cong B$.

Conditions (i) and (ii) are clear. For a proof of (v) we refer to [7] or [21]. The fact that if $G$ is totally projective then $p^{\alpha} G$ and $G / p^{\alpha} G$ are also is clear. For the other half of (iii) we remark that the basic extension theorem (1.2 below, proved in [7] and [21]) shows that if $p^{\alpha} G$ and $G / p^{\alpha} G$ are totally projective and if the Ulm invariants of $G$ are those of a totally projective group, then $G$ is totally projective. This is easy to see if $\alpha$ is a finite ordinal, and the rest of the proof can be given as in that of 5.1 below. An alternative proof is in $[3,81.9$ and 81(A)]. (iv) is an easy consequence of (v) and a suitable existence theory, and a proof of it is contained in our proof of 4.6 below. There are a number of other easily established properties of totally projective groups which we do not list because their generalizations for $K T$-modules and $S$-groups are either difficult or unknown. Condition (v) has a generalization which we will need, also essentially due to Hill, and stated in this form in [21].

Definition. If $G$ is a group whose torsion subgroup is a $p$-group, $A$ is a subgroup, and $A_{\alpha}=A \cap p^{\alpha} G$, we define

$$
I_{\alpha}(A)=\left(A_{\alpha}+p^{\alpha+1} G\right)[p] /\left(p^{\alpha+1} G\right)[p] .
$$

We regard $I_{\alpha}(A)$ as a subspace of the Ulm invariant $U_{\alpha}(G)$.

1.2. THEOREM ([7], [21]). If $G$ and $H$ are groups, $A$ and $B$ p-nice subgroups of $G$ and $H$ respectively such that $G / A$ and $H / B$ are totally projective p-groups, $\phi: A \rightarrow B$ an isomorphism such that, for all $\alpha, \phi\left(A_{\alpha}\right)=B_{\alpha}$, and if, for all $\alpha$, $U_{\alpha}(G) / I_{\alpha}(A) \cong U_{\alpha}(H) / I_{\alpha}(B)$, then $\phi$ extends to an isomorphism of $G$ onto $H$.

1.3. CoRollary. If $M$ and $N$ are $K T$-modules, $f(\alpha, M)=f(\alpha, N)$ for all ordinals $\alpha$, and $h(\lambda, M)=h(\lambda, N)$ for all limit ordinals $\lambda$, then $M \cong N$.

Proof. We write $M=T \oplus\left(\bigoplus_{i \in I} M_{i}\right)$, where $T$ is a totally projective $p$ - 
group and $M_{i}$ is a $\lambda(i)$-elementary $K T$-module. For each $i \in I$, choose $x_{i}$ to be a generator of $p^{\lambda(i)} M_{i}$ and let $X=\left\{x_{i}: i \in I\right\}$. We similarly choose a subset $Y=$ $\left\{y_{j}: j \in J\right\}$ of $N$. For a given ordinal $\lambda$, the number of elements of $I$ with $\lambda(i)=$ $\lambda$ is exactly $h(\lambda, M)$. There is, therefore, a bijective map $\phi: I \rightarrow J$ such that $\lambda(i)=\lambda(\phi(i))$ for all $i \in I$.

From the comments following the definition of $p$-nice subgroups, it is clear that the submodules $[X]$ and $[Y]$, generated by $X$ and $Y$, are $p$-nice submodules of $M$ and $N$, and it is clear by the construction that $M /[X]$ and $N /[Y]$ are totally projective $p$-groups. It is easily computed that for all $\alpha, I_{\alpha}([X])=0$ and $I_{\alpha}([Y])=0$. Since $X$ and $Y$ are free generators of the free modules $[X]$ and $[Y]$, $\phi$ defines an isomorphism $\phi^{\prime}:[X] \rightarrow[Y]$. It is now clear that all the hypotheses of 1.2 are satisfied, from which we conclude that $M \cong N$.

1.4. Lemma. If $0 \rightarrow A \rightarrow B \rightarrow C \rightarrow 0$ is an exact sequence of modules, then multiplication by $p$ in $B$ induces a homomorphism $\sigma: C[p] \rightarrow A / p A$. If we regard $A$ as a submodule of $B$ and $\nu: B \rightarrow C$ is the homomorphism of the sequence, then $\operatorname{ker}(\sigma)=\nu(B[p])$. For any ordinal $\alpha>0$, if $A \subseteq p^{\alpha} B$ then $\sigma$ is surjective and $C[p]=\operatorname{ker}(\sigma)+\left(p^{\beta} C\right)[p]$ for all $\beta<\alpha$. If $H$ is a submodule of $B$ maximal with respect to the property that $H \cap A=0$, then $v(H)[p]=\operatorname{ker}(\sigma)$.

Proof. We define $\sigma$ as follows: if $x \in C[p]$ choose a $y \in B$ such that $\nu(y)=x$ and let $\sigma(x)=p y+p A$. This is clearly well defined, and it is clear that $\operatorname{ker}(\sigma)=\nu(B[p])$. If $\alpha>0$, then $A \subseteq p^{\alpha} B$ if and only if for $\beta<\alpha$ and for any $x \in A$, there is a $y \in p^{\beta} B$ such that $p y=x$. This implies that $x+p A=\sigma(\nu(y))$ and $\nu(y) \in \nu\left(p^{\beta} B\right)[p] \subseteq\left(p^{\beta} C\right)[p]$, which proves all of the result but the last statement, which is trivial.

If $M$ is a $Z_{p}$-module and $N$ a submodule, we follow Nunke in saying that $N$ is $p^{\lambda}$-pure in $M$ if the corresponding extension $0 \rightarrow N \rightarrow M \rightarrow C \rightarrow 0$ is in $p^{\lambda} \operatorname{Ext}(C, N)$.

1.5. Lemma. Let $G$ be a p-group and $H$ a subgroup, $\lambda$ a limit ordinal, $\lambda>0$, and suppose that $p^{\lambda} G=0$. Then the following conditions are equivalent.

(i) $H$ is $\lambda$-dense and isotype.

(ii) $H$ is $p^{\lambda}$-pure and $G / H$ is divisible.

(iii) $H \cap p G=p H$ and, for all ordinals $\alpha<\lambda, G[p]=H[p]+\left(p^{\alpha} G\right)[p]$.

(iv) There is a $Z_{p}$-module $M$ and an imbedding $\phi: H \rightarrow M$ and $a$ surjective homomorphism $\psi: M \rightarrow G$ such that $\operatorname{ker}(\psi)=p^{\lambda} M, \phi(H)$ is $\lambda$-high in $M$, and $\psi \phi$ is the natural imbedding of $H$ into $G$.

(v) There is a $Z_{p}$-module $M$ and an imbedding $\phi: H \rightarrow M$ and a surjective homomorphism $\psi: M \rightarrow G$ such that $\operatorname{ker}(\psi)=p^{\lambda} M, p^{\lambda} M$ is free, $\phi(H)$ is the 
torsion submodule of $M$, and $\psi \phi$ is the natural imbedding of $H$ into $G$.

Proof. The equivalence of (i) and (iii) seems to be due to R. S. Pierce [20]. We show that (i) implies (iii). The first condition of (iii) is trivial. For the second, by $\lambda$-density, if $x \in G[p]$, there is a $y \in H$ and a $z \in p^{\alpha} G$ such that $x=$ $y-z$. It follows that $p y=p z \in p^{\alpha+1} G$, so, since $H$ is an isotype subgroup, $p y \in p^{\alpha+1} H$, so there is a $y^{\prime} \in p^{\alpha} H$ such that $p y=p y^{\prime}$. Hence, $x=\left(y+y^{\prime}\right)-$ $\left(z+y^{\prime}\right)$, where $y+y^{\prime} \in H[p]$ and $z+y^{\prime} \in\left(p^{\alpha} G\right)[p]$.

The equivalence of (ii) and (iii) is due to Nunke. (ii) implies (iii) by [16, 2.9]. Conversely, if (iii) holds, then since $p H=H \cap p G$, any element in $(G / H)[p]$ is in the image of $G[p]$, and hence in the image of $\left(p^{\alpha} G\right)[p]$ for any $\alpha<\lambda$. It follows that $G / H$ is divisible, from which (ii) follows, again by [16, 2.9].

That (iii) implies (v) seems to be due (in an equivalent form) to Kulikov [12]. For a proof see also [8, 1.7]. (The basic idea is to look at the sequence $0 \rightarrow H \rightarrow G \rightarrow D \rightarrow 0$ and an epimorphism $f: L \rightarrow D$, where $L$ is divisible and torsion-free and $\operatorname{Ker}(f)$ is free. The induced extension $0 \rightarrow H \rightarrow M \rightarrow$ $L \rightarrow 0$ works.) (v) implies (iv) trivially.

To complete the proof, we will show that (iv) implies (i). By [10, Theorem 2], $\phi(H)$ is an isotype submodule of $M$. We recall that if $G$ is a group and $N$ a subgroup, $H$ is $N$-high [9] in $G$ if $H \cap N=0$ and $H$ is maximal with respect to this property. It is easy to verify that this implies that the image of $N$ is essential in $G / H$, and if $N \subseteq p^{\omega} G$, it follows from this that $G / N$ is $p$-divisible. It is clear that if $H$ is $\lambda$-high in $M$, then for any $\alpha<\lambda, H \cap p^{\alpha} M$ is $\left(p^{\lambda} M\right)$-high in $p^{\alpha} M$. Since $\lambda$ is a limit ordinal, the elements of $p^{\lambda} M$ still have infinite height in $p^{\alpha} M$ for any $\alpha<\lambda$, which implies that $p^{\alpha} M /\left(\phi(H) \cap p^{\alpha} M\right)$ is divisible. Therefore, the image of $p^{\alpha} M$ in $M / \phi(H)$ is all of $M / \phi(H)$ (since it is a divisible submodule of $M / \phi(H)$ containing the image of $p^{\lambda} M$, an essential submodule). This implies that $M=\phi(H)+p^{\alpha} M$, which implies that $G=H+p^{\alpha} G$, since the map $\psi$ is surjective.

1.6. THEOREM. Let $G$ be a totally projective p-group of length $\lambda, \lambda$ a limit ordinal. Let $H$ and $K$ be two $\lambda$-dense, isotype subgroups of $G$. Then there is an automorphism $\sigma$ of $G$ such that $\sigma(H)=K$, if and only if $H[p]$ and $K[p]$ have the same codimension in $G[p]$.

Proof. The condition on $H$ and $K$ is clearly necessary.

By 1.5 , there are modules $M$ and $N$ and imbeddings $\phi: H \rightarrow M, \phi^{\prime}: K \rightarrow$ $N$, such that $p^{\lambda} M$ and $p^{\lambda} N$ are free, $\phi(H)$ and $\phi^{\prime}(K)$ are the torsion submodules of $M$ and $N$, and there are homomorphisms $\psi: M \rightarrow G$ and $\psi^{\prime}: N \rightarrow G$, satisfy- 
ing the conditions of $1.5(\mathrm{v})$. If $F=p^{\lambda} M$, then multiplication by $p$ induces an isomorphism $G[p] / H[p] \rightarrow F / p F$, by 1.4 , so that if $H[p]$ and $K[p]$ have the same codimension, then $p^{\lambda} M \cong p^{\lambda} N$, since they are free and of the same rank. The conditions of 1.2 are satisfied, so we get an isomorphism of $M$ onto $N$, which clearly carries $\phi(H)$ onto $\phi^{\prime}(K)$. The isomorphism between $M$ and $N$ induces an isomorphism $M / p^{\lambda} M \rightarrow N / p^{\lambda} N$, and since $\psi$ and $\psi^{\prime}$ can be used to identify both of these modules with $G$, we have an induced automorphism of $G$ with the desired properties.

Theorem 1.6 admits of a generalization, using the $\lambda$-Zippin property.

Definition. If $M$ is a module, $\lambda$ a limit ordinal, and $p^{\lambda} M=0$, then $M$ has the $\lambda$-Zippin property if for every triple $(G, H, f)$, where $G$ and $H$ are modules, $G / p^{\lambda} G \cong H / p^{\lambda} H \cong M$ and $f: p^{\lambda} G \rightarrow p^{\lambda} H$ is an isomorphism; there is an extension of $f$ to an isomorphism $f^{\prime}: G \rightarrow H$.

1.7. Lemma. If $G$ is an $S$-group or a KT-module, and $p^{\lambda} G=0$, then $G$ has the $\lambda$-Zippin property.

This is proved by Nunke in [18]. The proof of 1.6 immediately implies the following.

1.8. THEOREM. If $G$ is a p-group, $\lambda$ a limit ordinal, $p^{\lambda} G=0, G$ has the $\lambda$ Zippin property, $H$ and $K$ are $\lambda$-dense, isotype subgroups of $G$, and $\operatorname{dim}((G / H)[p])=\operatorname{dim}((G / K)[p])$, then there is an automorphism of $G$ taking $H$ onto $K$.

2. Cotorsion envelopes and the functors $K_{\lambda}$. In this section we will define a family of functors $K_{\lambda}$, where $\lambda$ is any ordinal. We will need the theory of cotorsion modules, which were introduced independently by Fuchs [2], Nunke [14] and Harrison [4]. We follow Fuchs in saying that a module $C$ is cotorsion if and only if $\operatorname{Ext}(F, C)=0$ for all torsion-free modules $F$. (It is sometimes required that a cotorsion module be reduced.) If we identify a module $M$ with $\operatorname{Hom}\left(Z_{p}, M\right)$, then we get a natural map $M \rightarrow \operatorname{Ext}\left(Q / Z_{p}, M\right)$, which is injective if $M$ is reduced. If $M$ is reduced, we let $c(M)=\operatorname{Ext}\left(Q / Z_{p}, M\right)$, and regard $M$ as contained in $c(M)$. We refer to Nunke [14] or Harrison [4] for the proofs that $c(M)$ is cotorsion and reduced and that $c(M) / M$ is torsion-free and divisible. This last implies easily that $M$ is isotype in $c(M)$, and that the torsion submodule of $c(M)$ is contained in $M$. We recall that a submodule $N$ of $M$ is pure in $M$ if $p^{n} N=N \cap p^{n} M$ for all $n, 0 \leqslant n<\omega$.

\subsection{Lemma. If $M$ is a reduced module and $N$ a submodule such that $M / N$} is reduced, then the sequence 


$$
0 \rightarrow c(N) \rightarrow c(M) \rightarrow c(M / N) \rightarrow 0
$$

is exact. If $M$ is cotorsion, then so is $N$. In particular, if $M$ is a reduced cotorsion module then so is $p^{\lambda} M$, for any ordinal $\lambda$.

Proof. The first follows from the exact sequence for Ext and the fact that $\operatorname{Hom}\left(Q / Z_{p}, M / N\right)=0$. For the second statement, we use the fact that a module $C$ is cotorsion if and only if $\operatorname{Ext}(Q, C)=0$ (since this implies that $\operatorname{Ext}(D, C)=$ 0 for any torsion-free divisible module $D$, and any torsion-free module can be imbedded in such a $D)$. If $\operatorname{Ext}(Q, M)=0$, then since $\operatorname{Hom}(Q, M / N)=0$, $\operatorname{Ext}(Q, N)=0$.

\subsection{Lemma. Let $M$ be a reduced module and $N$ a submodule such that} $M / N$ is torsion-free and divisible. Then (i) if $C$ is a reduced cotorsion module and $f: N \rightarrow C$ a homomorphism, there is a unique extension of $f$ to a homomorphism $g: M \rightarrow C$, (ii) if $f$ is injective and $C / f(N)$ is torsion-free, then $g$ will also be injective, and (iii) if $M$ is cotorsion then there is a natural isomorphism $M \cong c(N)$.

Proof. We have an exact sequence

$$
\operatorname{Hom}(M / N, C) \rightarrow \operatorname{Hom}(M, C) \rightarrow \operatorname{Hom}(N, C) \rightarrow \operatorname{Ext}(M / N, C) .
$$

The hypotheses imply that the extremal terms of this sequence vanish, which implies (i). For (ii), we remark that the kernel $K$ of $g$ will be isomorphic to a subgroup of $M / N$, and if $C / f(N)$ is torsion-free, then $M / N+K$ will also be torsion-free, so $K$ must be isomorphic to a pure subgroup of $M / N$. A pure subgroup of $M / N$ is divisible, and since $M$ has no divisible subgroups except $0, K=$ 0 . For (iii) we apply (i) in both directions to the imbeddings of $N$ into $M$ and $c(N)$.

A torsion-free module is cotorsion if and only if it is algebraically compact [11], which means that it is a direct sum of a divisible module and a module which is complete and Hausdorff in its $p$-adic topology. Part (iii) of 2.2 implies that if $M$ is a reduced torsion-free module, then $c(M)$ can be identified with the $p$-adic completion of $M$. We recall that if $A$ is a torsion-free, reduced module which is complete in its $p$-adic topology, and $\nu: A \rightarrow A / p A$ is the natural map, and if $X$ is a subset of $A$ which is taken by $\nu$ bijectively onto a $Z / p Z$-basis of $A / p A$, then the submodule $[X]$ generated by $X$ is free, with $X$ as a set of free generators, and $A$ is the $p$-adic completion of [X]. In particular, if $M$ is a module such that $p^{\lambda} M=0$, then $p^{\lambda} c(M)$ is complete, torsion-free module, since it is cotorsion by 2.1 and torsion-free (since $M \cap p^{\lambda} c(M)=p^{\lambda} M=0$ ). The above anal$y$ sis then motivates the following definition.

Definition. If $M$ is a $Z_{p}$-module, and $\lambda$ is an ordinal, we define 


$$
K_{\lambda}(M)=p^{\lambda} c\left(M / p^{\lambda} M\right) / p^{\lambda+1} c\left(M / p^{\lambda} M\right) .
$$

If we let $k(\lambda, M)$ be the $Z / p Z$ dimension of $K_{\lambda}(M)$, we obtain a new family of invariants indexed by ordinals. We remark that in [5] Harrison defined a new sequence of invariants which, in our terminology, were the invariants $h(\lambda, c(M))$. In general it is easy to verify that $h(\lambda, c(M))=h(\lambda, M)+k(\lambda, M)$, so our present invariants are more delicate than Harrison's for modules in general, but equivalent for $p$-groups.

Though we define $K_{\lambda}$ for any ordinal $\lambda$, it is clear that if $0<n<\omega$, then $K_{\lambda}$ and $K_{\lambda+n}$ are equivalent functors, so that for most purposes we can restrict ourselves to limit ordinals.

\subsection{Lemma. Let $M$ be a reduced $Z_{p}$-module and $\lambda>0$ a limit ordinal} which is not cofinal with $\omega$. Suppose that $M / p^{\lambda} M$ is a totally projective p-group and $p^{\lambda} M$ is torsion-free. Then there is a natural isomorphisms $p^{\lambda} M / p^{\lambda+1} M \rightarrow$ $K_{\lambda}(H)$ where $H$ is the torsion submodule of $M$.

Proof. $M / H$ is divisible and torsion-free, so by 2.2 , there is an imbedding $\phi: M \rightarrow c(H)$. Since $M / H$ is torsion-free and divisible, $c(H) / M$ is torsion-free and divisible, so $p^{\lambda} M$ is an isotype submodule of $p^{\lambda} c(H)$. Since $p^{\lambda} c(H)$ is reduced and torsion-free, it is complete in its $p$-adic topology, and if $L$ is the $p$-adic completion of the pure submodule $p^{\lambda} M$, then $L$ is a summand of $p^{\lambda} c(H)$ by [11, Theorem 23]. The quotient $c(H) / L$ is a reduced cotorsion module with $M / p^{\lambda} M$ as its torsion submodule. If $C=c(H) / L$ and $G$ is the image in $C$ of $M / p^{\lambda} M$, then $C / G$ is torsion-free and divisible, so we can identify $C$ with $c(G)$ by 2.2 . Since $G$ is totally projective, it is a direct sum of groups of smaller length, so by [16, 3.10], $p^{\lambda} c(G)=0$ (using the fact that $\lambda$ is not cofinal with $\omega$ ). Hence $L=$ $p^{\lambda} c(H)$, which gives the desired result.

It is clear that the functors $K_{\lambda}$ are additive in the sense that there is a natural isomorphism $K_{\lambda}(M \oplus N)=K_{\lambda}(M) \oplus K_{\lambda}(N)$. This additivity does not extend to infinite direct sums, as trivial examples show. However, if $\lambda$ is a limit ordinal which is not cofinal with $\omega$, Nunke has shown that $K_{\lambda}$ is strongly additive in this sense. We will not need this general result, since the special case which occurs when all of the modules involved are $S$-groups is quite easy.

3. Classification theorems. We classify dense isotype subgroups of totally projective $p$-groups up to isomorphism, and use this to classify $S$-groups. The groups classified in 3.1 actually are $S$-groups, but to prove this, one needs some considerations concerning admissible invariants which we postpone till $\S 4$. 
ordinal. If $\lambda$ is cofinal with $\omega$ then all $\lambda$-dense, isotype subgroups of $G$ are isomorphic to $G$. If $\lambda$ is not cofinal with $\omega$, then two $\lambda$-dense, isotype subgroups $H$ and $K$ are isomorphic if and only if $H[p]$ and $K[p]$ have the same codimension in $G[p]$.

Proof. If $\lambda$ is cofinal with $\omega$, then by Nunke's theorem $[16,4.4], p^{\lambda}$ Ext is hereditary, which implies that any $p^{\lambda}$-pure subgroup of $G$ is $p^{\lambda}$-projective. If $H$ is a $\lambda$-dense, isotype subgroup of $G$, then $H$ is $p^{\lambda}$-projective by 1.5 , which certainly implies that $H$ is $p^{\alpha}$-projective for any $\alpha>\lambda$. If $\alpha<\lambda$, then $H / p^{\alpha} H \cong$ $G / p^{\alpha} G$, so $H / p^{\alpha} H$ is $p^{\alpha}$-projective. This shows that $H$ is totally projective, and since its Ulm invariants are the same as those of $G, H \cong G$.

To show the second half of the theorem, we must show that if $H$ is a $\lambda$ dense, isotype subgroup of $G$ and is not cofinal with $\omega$, then the codimension of $H[p]$ in $G[p]$ is an isomorphism invariant of $H$, and the rest will follow by Theorem 1.6. Using part (v) of 1.5 , we construct a $Z_{p}$-module $M$ and a mapping $\psi$ : $H \rightarrow M$ taking $H$ isomorphically onto the torsion submodule of $M$. We also have a surjective mapping $\psi: M \rightarrow G$ with kernel $p^{\lambda} M$ such that $\psi \phi$ is the natural imbedding of $H$ into $G$. It follows, from 1.4, that there is a natural isomorphism $G[p] / H[p] \rightarrow p^{\lambda} M / p^{\lambda+1} M$, given by multiplication by $p$. Since $\lambda$ is not cofinal with $\omega, 2.3$ implies that $p^{\lambda} M / p^{\lambda+1} M \cong K_{\lambda}(H)$, which shows that the codimension of $H[p]$ in $G[p]$ is indeed an isomorphism invariant of $H$.

DEFinition. If $M$ is a module and $\lambda$ is a limit ordinal which is not cofinal with $\omega$, we define $k(\lambda, M)=\operatorname{dim}\left(K_{\lambda}(M)\right)$, where $K_{\lambda}(M)$ is regarded as a $Z / p Z$ vector space.

3.2. THEOREM. If $G$ and $H$ are $S$-groups, then $G \cong H$ if and only if for every ordinal $\alpha$ and every limit ordinal $\lambda$ which is not cofinal with $\omega, f(\alpha, G)=$ $f(\alpha, H)$ and $k(\lambda, G)=k(\lambda, H)$. Any $S$-group $G$ is a direct sum of a totally projective $p$-group and $\lambda$-elementary $S$-groups where the ordinals $\lambda$ are not cofinal with $\omega$. The number of $\lambda$-elementary summands in such a representation is exactly $k(\lambda, G)$. In particular, $G$ is totally projective if and only if $k(\lambda, G)=0$ for all limit ordinals $\lambda$ which are not cofinal with $\omega$.

Proof. We first remark that if $G$ is totally projective then $k(\lambda, G)=0$ for all limit ordinals $\lambda$ which are not cofinal with $\omega$, by $[16,3.10]$, since $G / p^{\lambda} G$ is a direct sum of groups of smaller length. We remark that this makes the last statement of the theorem a trivial consequence of the rest.

We next complete the proof of the second half of the theorem. Any $S$. group is a direct sum of a totally projective group and $\lambda$-elementary $S$-groups for various ordinals $\lambda$, by the definition. By 3.1 , if $\lambda$ is a limit ordinal which is co- 
final with $\omega$, then a $\lambda$-elementary $S$-group is totally projective. Therefore, any $S$-group is the direct sum of a totally projective group and $\lambda$-elementary $S$-groups, where the $\lambda$ 's may all be assumed to be not cofinal with $\omega$. If $\Lambda$ is the set of limit ordinals (not dofinal with $\omega$ ) for which summands appear, then we can write $G=T \oplus\left(\bigoplus_{\lambda \in \Lambda} G_{\lambda}\right)$ where each $G_{\lambda}$ is a direct sum of $\lambda$-elementary $S$-groups. Fixing $\lambda$, we write $G=T \oplus A \oplus G_{\lambda} \oplus B$, where $A$ is the direct sum of the $G_{\gamma}$ for $\gamma>\lambda$ and $B$ is the direct sum of the $G_{\gamma}$ for $\gamma<\lambda . k(\lambda, T)=0$ by the first remark of the proof. $k(\lambda, B)=0$, since $p^{\lambda} c(B)=0 . k(\lambda, A)=0$ since if $E$ is a $\gamma$-elementary $S$-group and $\lambda<\gamma$, then $E / p^{\lambda} E$ is totally projective. Hence $k(\lambda, G)=$ $k\left(\lambda, G_{\lambda}\right) . G_{\lambda}$ is the torsion submodule of a $K T$-module $M_{\lambda}$ such that $p^{\lambda} M_{\lambda}$ is a free module whose rank is the number of $\lambda$-elementary summands of $G_{\lambda}$. By 2.3, $k(\lambda, G)$ is equal to the rank of $p^{\lambda} M_{\lambda}$, which establishes the computation of $k(\lambda, G)$ stated in the second part of the theorem.

We turn now to the first part of the theorem. We have shown that an $S$ group $G$ can be regarded as the maximal torsion subgroup of a $K T$-module $M$, where $M$ is the direct sum of a totally projective $p$-group and $\lambda$-elementary $K T$ module, $\lambda>0$, where the ordinals $\lambda$ are not cofinal with $\omega$, and if this is done, then the number of $\lambda$-elementary summands of $M$ is just $k(\lambda, G)$. It is also $h(\lambda, M)$. We note also that the Ulm invariants of $M$ and $G$ agree, and that if $\lambda$ is a limit ordinal which is cofinal with $\omega$, then $h(\lambda, M)=0$. Hence we can imbed $G$ and $H$ as the torsion submodules of $K T$-modules $M$ and $N$ with the same invariants. By $1.3, M \cong N$, and since $G$ and $H$ are the maximal torsion submodules, $G \cong H$.

\section{Admissible sets of invariants.}

Definition. Let $f$ and $h$ be functions associating to each ordinal number a cardinal number, and such that for some ordinal $\gamma, f(\alpha)=h(\alpha)=0$ for all $\alpha$, $\alpha>\gamma$. Let

$$
\lambda(f, h)=\sup \{\alpha+1, f(\alpha) \neq 0 ; \alpha+\omega, h(\alpha) \neq 0\} .
$$

We say $(f, H)$ is an admissible pair if $h(\alpha)=0$ unless $\alpha$ is a limit ordinal, and if $\alpha$ is a limit ordinal with $\alpha+\omega<\lambda(f, h)$, and $0 \leqslant n<\omega$, then

$$
\sum_{\alpha+n \leqslant \beta<\alpha+\omega} f(\beta) \geqslant \sum_{\alpha+\omega \leqslant \beta<\lambda(f, h)}(f(\beta)+h(\beta)) .
$$

4.1. THEOREM. If $f$ and $h$ are functions associating to each ordinal a cardinal number, then there is a KT-module $M$ such that $f(\alpha, M)=f(\alpha)$ and $h(\beta, M)=$ $h(\beta)$ for all ordinals $\alpha$ and all limit ordinals $\beta$ if and only if $(f, h)$ is an admissible pair. 
Before proving this theorem, we investigate a large class of modules whose Ulm invariants satisfy the above inequalities. The next lemma is motivated by a method used by Nunke in [15, pp. 167-168]. For any module $A$, we let $\#(A)$ be the smallest cardinal number which is the cardinality of a set of generators of $A$. (For the countable ring $Z_{p}$, this number is just the cardinality of $A$, except when $A$ is finite, but the version presented here is valid over any discrete valuation ring.)

Definition. A module $M$ is said to be in the class $\Theta$ if and only if for every submodule $A$ of $M, \#(A)=\#(A / p A)$.

4.2. Lemma. If $M_{i}, i \in I$, are in the class $\Theta$ then $\bigoplus_{i \in I} M_{i}$ is also in $\Theta$. If $M$ is a module and $C$ a submodule which is finitely generated, and if $M / C$ is in $\Theta$, then $M$ is in $\Theta$.

Proof. In the first case, let $A$ be a submodule of $\bigoplus_{i \in I} M_{i}$. Let $X$ be a subset of $A$ which is taken bijectively onto a basis of $A / p A$ under the natural map, and let $B=[X]$. Clearly, $\#(B)=\#(A / p A)$. Let $\pi_{i}$ be the projection of $M$ onto the summand $M_{i}$. Since $A=B+p A, \pi_{i}(A)=\pi_{i}(B)+p_{\pi_{i}}(A)$, and since $M_{i} \in$

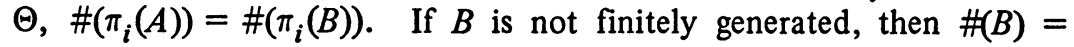
$\Sigma_{i \in I} \#\left(\pi_{i}(B)\right)$, and $A$ is also infinitely generated, so $\#(A)=\Sigma_{i \in I} \#\left(\pi_{i}(A)\right)$ and the result follows. If $B$ is finitely generated, then since $\pi_{i}(A) / \pi_{i}(B)$ is divisible, and since it is clear that an element of $\Theta$ cannot have a divisible submodule, $\pi_{i}(A)=0$ for all but finitely many $i$, since the same holds for $B$. Since each $\pi_{i}(A)$ is finitely generated, it follows that $A$ must be finitely generated, in which case a standard computation shows that $\#(A)=\#(A / p A)$.

For the second statement, we may restrict ourselves to infinitely generated submodules of $M$ entirely. If $\phi: M \rightarrow M / C$ is the natural map, and $A$ is a submodule of $M$ which is not finitely generated, then $\#(A)=\#(\phi(A))=$ $\#(\phi(A) / p \phi(A)) \leqslant \#(A / p A)$, from which the result follows.

4.3. Lemma. If $M \in \Theta$ and for any ordinal $\alpha$ and limit ordinal $\beta$ we define $f(\alpha)=f(\alpha, M)$ and $h(\beta)=h(\beta, M)$, and set $h(\alpha)=0$ for any ordinal $\alpha$ which is not a limit ordinal, then $(f, h)$ is an admissible pair.

Proof. All that we need to prove is the inequality (*) of the definition. If $\alpha$ is a limit ordinal, we can write $p^{\alpha} M=B \oplus A$ where $p^{n} B=0$ and $A$ has no summands which are cyclic of order less than $p^{n+1}$. Let $\nu: A \rightarrow A / p^{\alpha+\omega} M$ be the natural map and let $N$ be the submodule consisting of all elements of $A$ which are mapped into the torsion submodule of $A / p^{\alpha+\omega} M$. We note that $p^{\alpha+\omega} M=$ $p^{\omega} A=p^{\omega} N$, and if $k<\omega$, we compute the Ulm invariants $f(k, N)=0, k<n$, and $f(k, N)=f(\alpha+k), k \geqslant n$. From this, it is clear that 


$$
\#(N / p N)=\sum_{\alpha+n<\beta<\alpha+\omega} f(\beta) .
$$

Since $N \in \Theta$ and $p^{\alpha+\omega} M \subseteq N$, we can prove the inequality by finding a submodule $C$ of $p^{\alpha+\omega} M$ such that

$$
\#(C)=\sum_{\alpha+\omega<\beta<\lambda}(f(\beta)+h(\beta))
$$

(since $\#(C) \leqslant \#(N)=\#(N / p N)$ ). (Here, $\lambda$ is the length of $M$.)

If $\gamma$ is a limit ordinal and $B_{\gamma}$ is a basic submodule of $p^{\gamma} M$ then

$$
\#\left(B_{\gamma}\right)=\sum_{\gamma \leqslant \beta<\gamma+\omega}(f(\beta)+h(\beta)) .
$$

Since $B_{\gamma} \cap p^{\gamma+\omega} M=0$, the submodules $B_{\gamma}$ of $M$ are independent, and if we let $C=\bigoplus B_{\gamma}$, where the direct sum ranges over all limit ordinals $\gamma, \alpha+\omega \leqslant \gamma<\lambda$, then $C$ has the desired properties.

4.4. Lemma. If $G$ is a p-group of length $\alpha$, there is a $Z_{p}$-module $M$ such that $p^{\alpha} M \cong Z_{p}$ and $M / p^{\alpha} M \cong G$.

This is a special case of a well-known existence theorem, first proved by Pierce (unpublished); see [20], [18, Theorem 1.6].

Proof OF THEOREM 4.1. The existence proof is by induction on $\lambda=$ $\lambda(f, h)$. We distinguish two cases: (i) when there is a limit ordinal $\gamma$ such that $\lambda=\gamma+\omega$ or $\lambda=\gamma+n$ for some positive integer $n$, and (ii) when no such $\gamma$ exists. Case (ii) is easy. By a standard infinite combinatorial argument, we can find functions $f_{i}$ and $h_{i}(i \in I)$ such that $f=\Sigma_{i \in I} f_{i}, h=\Sigma_{i \in I} h_{i}$, and for each $i \in I$, $\left(f_{i}, h_{i}\right)$ is an admissible pair with $\lambda\left(f_{i}, h_{i}\right)<\gamma$. By induction, there are $K T$-modules $M_{i}(i \in I)$ with invariants given by the functions $\left(f_{i}, h_{i}\right)$, and $M=\bigoplus_{i \in I} M_{i}$ corresponds to the pair $(f, h)$.

In case (i), we use a similar combinatorial argument to write $f$ and $h$ as sums of functions $f_{i}$ and $h_{i}(i \in I)$, such that each pair $\left(f_{i}, h_{i}\right)$ is admissible and we impose the additional restriction that for each $i \in I$,

$$
\sum_{\gamma<\alpha<\gamma+\omega}\left(f_{i}(\alpha)+h_{i}(\alpha)\right)=1 .
$$

By induction, there are $K T$-modules $H_{i}, i \in I$, such that $p^{\gamma} H_{i}=0$ and such that for $\alpha<\gamma, f\left(\alpha, H_{i}\right)=f_{i}(\alpha)$; and for limit ordinals $\beta, \beta<\gamma, h\left(\beta, H_{i}\right)=h_{i}(\beta)$. Let $C_{i}$ be a cyclic module, $C_{i} \cong Z_{p}$ if $h_{i}(\gamma) \neq 0$ and $C_{i} \cong Z / p^{n+1} Z$ if $f_{i}(\gamma+n) \neq$ 0 . By 4.4, there are $K T$. -modules $M_{i}$ such that $p^{\gamma} M_{i} \cong C_{i}$ and $M_{i} / p^{\gamma} M_{i} \cong H_{i}$. The module $M=\bigoplus_{i \in I} M_{i}$ now satisfies our conditions.

All of the modules produced in the existence part of the proof of 4.1 are in 
$\Theta$, by 4.2 . If $M$ is any $K T$-module, we can get from this existence proof a $K T$ module $N$ in $\Theta$ such that the invariants of $N$ and $N \oplus M$ agree, from which it follows (from 1.3) that $N \cong N \oplus M$. Since a summand of an element of $\Theta$ is in $\Theta$, this shows that any $K T$-module is in $\Theta$, thus (with another reference to 4.3 ) completing the proof of 4.1 .

4.5. Corollary . Let $\mathrm{C}$ be a class of modules containing the KT-modules, and such that the direct sum of two modules in $\mathrm{C}$ is in $\mathrm{C}$. Suppose that for all pairs of elements $M$ and $N$ of $C, M \cong N$ if for all ordinals $\alpha$ and all limit ordinals $\lambda, f(\alpha, M)=f(\alpha, N)$ and $h(\lambda, M)=h(\lambda, N)$. Then $C$ coincides with the class of $K T$-modules.

Proof. If $M$ is in $C$ there is a $K T$-module $N$ such that the invariants of $N$ and $N \oplus M$ are the same. It follows that $N \oplus M \cong N$, so that $M$ is a summand of a $K T$-module, and hence in $\Theta . M$ therefore has the same invariants as some $K T$-module $K$, from which it follows by hypothesis that $M \cong K$, thus proving the result.

4.6. Corollary. Let $\mathrm{C}$ be a class of $Z_{p}$-modules such that

(i) any module isomorphic to an element of $\mathrm{C}$ is in $\mathrm{C}$;

(ii) a direct sum of modules in $C$ is in $C$;

(iii) if for some ordinal $\alpha, p^{\alpha} G$ and $G / p^{\alpha} G$ are in $C$, then $G \in C$.

Then (a) if $Z / p Z$ is in $C, C$ contains all totally projective p-groups, and (b) if $Z_{p}$ is in $C, C$ contains all $K T$-modules.

This follows from the proof of 4.1. Notice that in (iii), if we had restricted $p^{\alpha} G$ to be cyclic of order $p$, we still would get all totally projective $p$-groups. Part (a) was proved by Parker and Walker in [19].

4.7. THEOREM. If $G$ is a p-group which can be imbedded in a totally projective group, then there is a totally projective p-group $H$ such that for all $\alpha$, $f(\alpha, G)=f(\alpha, H)$.

Proof. This is an immediate consequence of 4.1, 4.3, and the fact that all totally projective $p$-groups are in $\Theta$ (which follows from the existence proof in 4.1).

4.8. THEOREM. Let $f$ and $k$ be two functions associating to each ordinal a cardinal and such that there is some ordinal $\tau$ such that $f(\alpha)$ and $k(\alpha)$ vanish for $\alpha>\tau$. Suppose also that for any ordinal $\alpha, k(\alpha)=0$ unless $\alpha$ is a limit ordinal which is not cofinal with $\omega, \alpha>0$. Then there is an S-group $G$ such that $f(\alpha, G)=f(\alpha)$ and $k(\lambda, G)=k(\lambda)$ for all ordinals $\alpha$ and all limit ordinals $\lambda$ which 
are not cofinal with $\omega$, if and only if for any limit ordinal and integer $n, 0 \leqslant$ $n<\omega$,

$$
\sum_{\gamma+n \leqslant \beta<\gamma+\omega} f(\beta) \geqslant \sum_{\gamma+\omega \leqslant \beta<\tau}(f(\beta)+k(\beta)) .
$$

Proof. By the argument used in the proof of 3.2, any $S$-group is the torsion submodule of a $K T$-module $M$ such that $f(\alpha, M)=f(\alpha, G)$ for all ordinals $\alpha$, $h(\lambda, M)=0$ for any limit ordinal $\lambda$ which is cofinal with $\omega$, and $h(\lambda, M)=$ $k(\lambda, G)$ for any limit ordinal which is not cofinal with $\omega$. 4.1 therefore implies that the above inequality must hold. Conversely, if the above inequality holds, then there is a $K T$-module $M$ with these invariants, and its torsion submodule is the desired $G$.

\section{Closure properties of the classes of $S$-groups and $K T$-modules.}

5.1. THEOREM. If $M$ is a $Z_{p}$-module and $\delta$ an ordinal, then $M$ is a KT-module if and only if $p^{\delta} M$ and $M / p^{\delta} M$ are both $K T$-modules.

Proof. If $M$ is a $K T$-module, it is clear from the definition and the corresponding property for totally projective groups that $p^{\delta} M$ and $M / p^{\delta} M$ are $K T$-modules.

For the converse, we first remark that we may assume that $\delta$ is a limit ordinal. This is because we already know the corresponding result for totally projective $p$-groups, which makes it clear from the definition that if $M$ is a module and $p^{n} M$ is a $K T$-module, then so is $M$.

We next show that the invariants of $M$ are those of some $K T$-module. Let $\sigma:\left(M / p^{\delta} M\right)[p] \rightarrow p^{\delta} M / p^{\delta+1} M$ be the surjective homomorphism defined in Lemma 1.4. Let $L$ be a subgroup of $\left(M / p^{\delta} M\right)[p]$ which is mapped isomorphically onto $p^{\delta} M / p^{\delta+1} M$ by $\sigma$. Clearly, since $p^{\delta} M \in \Theta$,

$$
\#(L)=\sum_{\delta<\beta<\lambda}(f(\beta, M)+h(\beta, M))
$$

where $\lambda$ is the length of $M$. We wish to check the inequality (*) for a limit ordinal $\alpha, \alpha<\delta$ (since for other limit ordinals, the result is clear). For every limit ordinal $\gamma$ such that $\alpha<\gamma<\delta$, let $B_{\gamma}$ be a basic submodule of $p^{\gamma} M$ and $B_{\gamma}^{\prime}$ the image of $B_{\gamma}$ in $M / p^{\delta} M$. As in the proof of 4.3, if $C$ is the direct sum of the submodules $B_{\gamma}^{\prime}$, then

$$
\#(C)=\sum_{\alpha+\omega \leqslant \beta<\delta}(f(\beta, M)+h(\beta, M)) .
$$

Since $C \cap L=0$ by construction (this is why we did not choose the modules $B_{\gamma}^{\prime}$ as arbitrary basic submodules of $\left.p^{\gamma}\left(M / p^{\delta} M\right)\right)$, as in the proof of 4.3, 


$$
\#(C)+\#(L) \leqslant \sum_{\alpha+n \leqslant \beta<\alpha+\omega} f(\beta, M) .
$$

Putting these inequalities together, we obtain condition (*).

From this we know that there is a $K T$-module $N$ with the same invariants as $M$. Since $p^{\delta} N$ and $p^{\delta} M$ are $K T$-modules with the same invariants, there is an isomorphism $f: p^{\delta} M \rightarrow p^{\delta} N$. Since $K T$-modules of length $\delta$ have the $\delta$-Zippin property (1.7), $f$ extends to an isomorphism of $M$ onto $N$, proving the result.

5.2. THEOREM. If $\alpha$ is an ordinal and $G$ a group, then $G$ is an $S$-group if and only if $p^{\alpha} G$ and $G / p^{\alpha} G$ are S-groups.

Proof. If $G$ is an $S$-group then $G$ is the torsion subgroup of a $K T$-module M. $p^{\alpha} G$ is the torsion subgroup of the $K T$-module $p^{\alpha} M$, and therefore is an $S$ group. If $G$ is a $\lambda$-elementary $S$-group, then if $\alpha \geqslant \lambda, G / p^{\alpha} G=G$, and is therefore an $S$-group, while if $\alpha<\lambda, G / p^{\alpha} G$ is totally projective. Since any $S$-group, $G$, is a direct sum of $\lambda$-elementary $S$-groups and a totally projective group, it follows that $G / p^{\alpha} G$ is an $S$-group.

We suppose now that $G$ is a $p$-group, and that $p^{\alpha} G$ and $G / p^{\alpha} G$ are $S$-groups. Write $\alpha=\delta+n$, where $\delta$ is a limit ordinal. If $\phi: p^{\alpha} G \rightarrow M$ is an imbedding of $p^{\alpha} G$ as the torsion subgroup of a $K T$-module $M$ (in which it may be assumed that $M / p^{\alpha} G$ is divisible), then there is a module $M^{\prime}$ and an imbedding $\phi^{\prime}: p^{\delta} G \rightarrow M^{\prime}$ such that $p^{n} M^{\prime}=M$. By $5.1, M^{\prime}$ is a $K T$-module, and by construction, $p^{\delta} G$ is its torsion submodule. We therefore need only show that $G$ is an $S$-group given that $p^{\delta} G$ and $G / p^{\delta} G$ are $S$-groups, where $\delta$ is a limit ordinal.

By the same argument as used in the proof of 5.1, the invariants of $G$ are those of an $S$-group $H . \quad p^{\delta} G$ and $p^{\delta} H$ are therefore isomorphic $S$-groups and there is an isomorphism $\phi: p^{\delta} G \rightarrow p^{\delta} H$. By 1.7 , this extends to an isomorphism of $G$ onto $H$, proving the result.

5.3. THEOREM. If $G$ is an $S$-group and $\lambda$ a limit ordinal, a $\lambda$-high subgroup of $G$ is also an $S$-group. If $G$ is an $S$-group, $\lambda$ a limit ordinal and $p^{\lambda} G=0$, then a $\lambda$-dense, isotype subgroup of $G$ is an $S$-group.

Proof. By 1.5 , the first stated result is a consequence of the second. We assume, then, that $p^{\lambda} G=0$ and that $H$ is a $\lambda$-dense, isotype subgroup. By 1.5 , there is a $Z_{p}$-module $M$ and maps $\phi: H \rightarrow M, \psi: M \rightarrow G$ such that $\phi$ imbeds $H$ as the torsion submodule of $M, \operatorname{ker}(\psi)=p^{\lambda} M, p^{\lambda} M$ is free, $\psi$ is surjective, and $\psi \phi$ is the natural imbedding of $H$ into $G$.

We can imbed $G$ into a $K T$-module $M^{\prime}$ as its torsion submodule, by a map $f: G \rightarrow M^{\prime}$. Since the map $\operatorname{Ext}\left(M^{\prime}, p^{\lambda} M\right) \rightarrow \operatorname{Ext}\left(G, p^{\lambda} M\right)$ is surjective, $M$ is a submodule of a module $M^{\prime \prime}$ such that the map $\psi: M \rightarrow G$ extends to a surjective 
map $\psi^{\prime}: M^{\prime \prime} \rightarrow M^{\prime}$ with kernel $p^{\lambda} M$. Clearly, $p^{\lambda} M^{\prime \prime}$ is free and $M^{\prime \prime} / p^{\lambda} M^{\prime \prime} \cong$ $M^{\prime} / p^{\lambda} M^{\prime}$, so, by $5.1, M^{\prime \prime}$ is a $K T$-module. By construction, it is clear that $\phi$ imbeds $H$ as the torsion submodule of $M^{\prime \prime}$, so (by definition) $H$ is an $S$-group.

\section{Unsolved problems.}

Problem 1. Is a summand of an $S$-group necessarily an $S$-group?

Problem 2. If not, can summands of $S$-groups be classified by the same invariants?

Problem 3. Is there a class $C$ of reduced $p$-groups such that the direct sum of two groups in $C$ is in $C$, such that $C$ properly contains the class of $S$-groups, and such that the groups in $C$ can be classified by the same invariants?

Before Ulm's theorem was proved for totally projective groups, Walker showed [19] that if this could be done, Ulm's invariants could not be used to classify the elements of any larger class of reduced $p$-groups closed with respect to finite direct sums. His argument shows that any group in such a class $C$ (as described in Problem 3) would be a summand of an $S$-group. An affirmative answer to Problem 1 would therefore imply a negative answer to Problem 3, but possibly Problem 3 could be answered independently (compare 4.5 above). In [23], Wick gives a "projective" characterization of summands of $S$-groups.

Problem 4. Is there a reasonable structure theory for a larger class of groups which are isotype subgroups of totally projective groups?

Hill showed [6] that an isotype subgroup of a totally projective group of countable length is again totally projective. However, C. Megibben has pointed out to the author that an isotype subgroup of a direct sum of countable $p$-groups need not be an $S$-group. (This is easily obtained from an example in the last paragraph of [13, p. 109], using the fact that if $G$ is an $S$-group, then $L_{\Omega} G / G$ is divisible.)

Problem 5. Is there a reasonable structure theory for the groups which arise as $\operatorname{Tor}(A, B)$, when $A$ and $B$ are totally projective?

Nunke showed [14] that if $A$ and $B$ are totally projective of countable length, then $\operatorname{Tor}(A, B)$ is totally projective, but Wick [23] shows that there are totally projective groups $A$ and $B$ such that $\operatorname{Tor}(A, B)$ is not an $S$-group.

Problem 6. If $G$ is totally projective and $H$ a subgroup such that $(G / H)[p]$ is finite, is $H$ necessarily an $S$-group?

Edington [1] gives necessary and sufficient conditions, in this situation, for $H$ to be totally projective.

If $\lambda$ is a limit ordinal and $G$ is a $p$-group with $p^{\lambda} G=0$, we say $G$ has the $\lambda$-Zippin property if for all pairs $H$ and $K$ of $p$-groups such that $H / p^{\lambda} H \cong$ $K / p^{\lambda} K \cong G$ and all isomorphisms $f: p^{\lambda} H \rightarrow p^{\lambda} K$, there is an isomorphism $\phi$ : 
$H \rightarrow K$ whose restriction to $p^{\lambda} H$ is $f$. Nunke shows [18] that if $G$ is an $S$-group and $p^{\lambda} G=0$, then $G$ has the $\lambda$-Zippin property. The converse fails in a nontrivial way.

Problem 7. If $G$ is a $p$-group and for all limit ordinals $\lambda, G / p^{\lambda} G$ has the $\lambda$ Zippin property, is $G$ necessarily an $S$-group?

If $u$ is a function associating to each nonnegative integer an ordinal or $\infty$, and $G$ is a $p$-group, we define $u G=\left\{x \in G\right.$ : for all $\left.n \geqslant 0, p^{n} x \in p^{u(n)} G\right\}$. Kaplansky showed in [11] that if $G$ is a reduced countable $p$-group, then the fully invariant subgroups of $G$ are exactly the subgroups $u G$ obtained in this way. The methods now available make it trivial to extend this result to totally projective $p$-groups. L. Fuchs and E. A. Walker (unpublished) have shown that if $G$ is totally projective and $u$ is such a function, then $u G$ and $G / u G$ are again totally projective.

Problem 8. If $G$ is an $S$-group, are all fully invariant subgroups of the form $u G$ ?

Problem 9. If $G$ is an $S$-group, and $u$ is one of the above functions, are $u G$ and $G / u G$ necessarily $S$-groups?

Problem 10. Let $G$ and $H$ be reduced $p$-groups and $\phi: G \rightarrow H$ a homomorphism such that the induced maps $U_{\alpha}(G) \rightarrow U_{\alpha}(H)$ and $K_{\lambda}(G) \rightarrow K_{\lambda}(H)$ are isomorphisms for all ordinals $\alpha$ and all limit ordinals $\lambda$. Is $\phi$ necessarily an isomorphism?

The answer to Problem 10 is yes for groups with no elements of infinite height.

\section{REFERENCES}

1. B. Edington, Isomorphism invariants in quotient categories of Abelian groups, Thesis, New Mexico State University, 1972.

2. L. Fuchs, Notes on A belian groups. II, Acta Math. Acad. Sci. Hungar. 11 (1960), 117125. MR 23 \#A3175.

3. - Infinite Abelian groups. Vol. II, Academic Press, New York, 1973.

4. D. K. Harrison, Infinite A belian groups and homological methods, Ann. of Math. (2) 69 (1959), 366-391; Correction, ibid. (2) 71 (1960), 197. MR 21 \#3481; 23 \#A206.

5. - On the structure of Ext, Topics in Abelian Groups (Proc. Sympos., New Mexico State Univ., 1962), Scott, Foresman, Chicago, Ill., 1963, pp. 195-210. MR 39 \#4253.

6. P. D. Hill, Isotype subgroups of direct sums of countable groups, Illinois J. Math. 13 (1969), 281-290. MR 39 \#1550.

7. P. D. Hill, On the classification of Abelian groups, Mimeographed notes, 1968.

8. P. D. Hill and C. Megibben, On direct sums of countable groups and generalizations, Studies on Abelian Groups (Sympos., Montpellier, 1967), Springer, Berlin, 1968, pp. 183-206. MR 39 \#4270.

9. J. M. Irwin and E. A. Walker, On $N$-high subgroups of A belian groups, Pacific J. Math. 11 (1961), 1363-1374. MR 25 \#119a.

10. - On isotype subgroups of Abelian groups, Bull. Soc. Math. France 89 (1961), 45 1-460. MR 26 \#5054.

11. I. Kaplansky, Infinite A belian groups, rev. ed., Univ. of Michigan Press, Ann Arbor, Mich., 1969. MR 38 \#2208. 
12. L. Ya. Kulikov, Generalized primary groups. I, II, Trudy Moskov. Mat. Obšc. 1 (1952), 247-326; ibid. 2 (1953), 85-167. (Russian) MR 14, 132; 15, 9.

13. C. K. Megibben, On $p^{\alpha}$-high injectives, Math. Z. 122 (1971), 104-110. MR 45 \#8726.

14. R. J. Nunke, Modules of extensions over Dedekind rings, Illinois J. Math. 3 (1959), 222-241. MR 21 \# 1329.

15. - Purity and subfunctors of the identity, Topics in Abelian Groups (Proc. Sympos., New Mexico State Univ., 1962), Scott, Foresman, Chicago, Ill., 1963, pp. 121-171. MR 30 \#156.

16. - Homology and direct sums of countable abelian groups, Math. Z. 101 (1967), 182-212. MR 36 \#1538.

17. - On the structure of Tor II, Pacific J. Math. 22 (1967), 453-464. MR 35 \#5508.

18. - Uniquely elongating modules, Proc. Conf. on Algebraic Groups (November 1972), Symposia Mathematica, vol. 12, Academic Press, London, 1974, pp. 315-330.

19. L. D. Parker and E. A. Walker, An extension of the Ulm-Kolettis theorem, Studies on Abelian Groups (Sympos., Montpellier, 1967), Springer, Berlin, 1968, pp. 309-325. MR 42 \#367.

20. R. S. Pierce, Abelian Group Seminar Notes, New Mexico State University, 1968.

21. E. A. Walker, Ulm's theorem for totally projective groups, Proc. Amer. Math. Soc. 37 (1973), 387-392. MR 47 \#367.

22. R. B. Warfield, Jr., Classification theorems for p-groups and modules over a discrete valuation ring, Bull. Amer. Math. Soc. 78 (1972), 88-92. MR 45 \#378.

23. B. Wick, Classification theorems for infinite Abelian groups, Thesis, University of Washington, 1972.

DEPARTMENT OF MATHEMATICS, UNIVERSITY OF WASHINGTON, SEATTLE, WASHINGTON 98195 